\title{
EXPERIMENTAL AND COMPUTATIONAL STUDIES OF ACTIVE FLOW CONTROL ON A MODEL TRUCK-TRAILER
}

\author{
Mohammad EL-ALTI ${ }^{1,2}$, Valery CHERNORAY ${ }^{1}$, Mohsen JAHANMIRI ${ }^{1,3}$ and Lars DAVIDSON ${ }^{1}$
}

\begin{abstract}
Active flow control is probably the most challenging research area in vehicle aerodynamics. Being able to manipulate a flow field in order to achieve desired results beneficial to engineering is the only way to meet today's demands for competitive and efficient solutions in the automotive industry. The current work studies the flow control on a semi detailed model truck by using detached-eddy simulations and wind tunnel experiments aiming at reducing the aerodynamic drag. This study combines both passive and active flow control applied on the rear end of the trailer. An indigenous fluidic actuator (loudspeaker in cavity with slots) is used as a synthetic jet in the experiment. Both experiments and computations demonstrate that the active flow control works successfully and results in flow reattachment to the flaps. The numerical simulations show that the drag coefficient, $C_{D}$, decreased by $3.9 \%$ when AFC was activated compared to the baseline case without flaps. The corresponding decrease when AFC was deactivated (with flaps) was only $0.7 \%$. The experimental results show a decrease of $C_{D}$ by $3.1 \%$ for the case with activated AFC compared to the baseline case. When AFC was deactivated the corresponding decrease in $C_{D}$ was $1.8 \%$. A detailed flow analysis made in computations and experiments is used to explain these results.
\end{abstract}

\section{INTRODUCTION}

Active flow control (AFC) is a fast growing multi-disciplinary science and technology aimed at altering a natural flow state or development path into a more desired state (or path). Flow control research dates back to the discovery of the boundary layer by Prandtl [1] at the turn of the 20th century. Flow control was extensively studied and applied in the period leading up to and during World War II, as well as in the Cold War era, although primarily in military-related flow systems. A comprehensive review and analysis was provided by Lachman [2] and more recently by Gad-el-Hak et al. [3], Gad-el-Hak [4]. All known flow control efforts preceding the pioneering work of Schubauer and Skramstad [5] used steady-state tools and mechanisms for flow management. These are of inherently marginal power efficiency, and therefore limited the implementation of the resulting systems in operational applications. Unsteady flow control using periodic excitation that exploits natural flow instability phenomena such as control of flow separation has the potential to overcome the efficiency barrier.

\footnotetext{
${ }^{1}$ Division of Fluid Dynamics, Department of Applied Mechanics, Chalmers University of Technology SE-412 96 Göteborg, Sweden

${ }^{2}$ Corresponding author, email address: mohammad.el-alti@chalmers.se

${ }^{3}$ Dept. of Mech. and Aerospace Engineering, Shiraz University of Technology Shiraz, Iran
} 
As an example, Seifert et al. [6] showed that separation control using periodic addition of momentum, at a reduced frequency slightly higher than the natural vortex shedding frequency, can save $90-99 \%$ of the momentum required to obtain similar gains in performance using steady blowing.

The major contribution to drag on a truck is caused by the wake behind the trailer. The flow separation at the aft of the trailer results in a large low pressure region that increases the pressure difference between the front and back. An effective way to decrease the size of the separation zone and increase the base pressure is to use active and passive flow control techniques (AFC and PFC). Increasing fuel prices and harder environmental restrictions are the main driving forces in the development of new techniques that improve the aerodynamics of truck-trailers.

In the current work, the flow control on a semi detailed model truck is studied by detached-eddy simulations and wind tunnel experiments aiming at reducing the aerodynamic drag. This study combines both passive and active flow control applied to the rear end of the trailer. The idea is to suppress and decrease the size of the wake by attaching the separated flow onto inclined flaps mounted at the trailer rear end (sides and roof) and thus to increase the base pressure and reduce the overall drag. This study is a continuation of earlier work [7-9]. In previous studies the computations were performed using large eddy simulations on simplified truck models with AFC/PFC, and experiments were done on a full-scale Volvo truck-trailer. These efforts showed good possibilities to reduce the drag and thus the fuel consumption of truck-trailers. This project was carried out as a bachelor thesis supervised by the authors [10].

\section{The Model TRUCK AND CONFigurations}

The model truck is a 1:10 scale model of the Volvo FH16 that has a fully detailed cab and simplified underbody and wheel housing; the cooling flow is also closed. The trailer has a realistic geometry with simplified underbody. The CAD model was created in two separate main parts, tractor and trailer. The tractor geometry was simplified using CAE software, ANSA. Much effort was made to make the model well sealed both for the computations and for the SLS rapid prototyping machine. The trailer CAD was drawn in CATIA, which has the possibility to easily construct the flaps and actuation details and to export to ANSA or the SLS machine. Most of the trailer was made of Medium Density Fiberboard (MDF) material, however and was not printed in the SLS machine. The flaps and the box-shaped rear end were made to be easily interchangeable as modules connected with rails to the rear end of the trailer. The geometrically complicated parts such as the flap modules and the tractor, were printed. Three different configurations are studied: a trailer without flaps (baseline case), with flaps (PFC case) and with flaps and AFC (AFC case).

Several transitional ducts were made in the flap modules which smoothly guide the flow from the loudspeaker to the actuator slots, see Figure 2. The base pressure is measured via pressure tubes that are connected to integrated pressure channels in the interchangeable modules. The pressure was measured in 12 positions for the baseline case and 16 positions for the PFC/AFC case. The positions are at one half side of the trailer base assuming symmetry. Figure 3 shows the distribution of the pressure locations. For the module with flaps, ten positions are on the flaps surfaces and six are on the trailer base. 

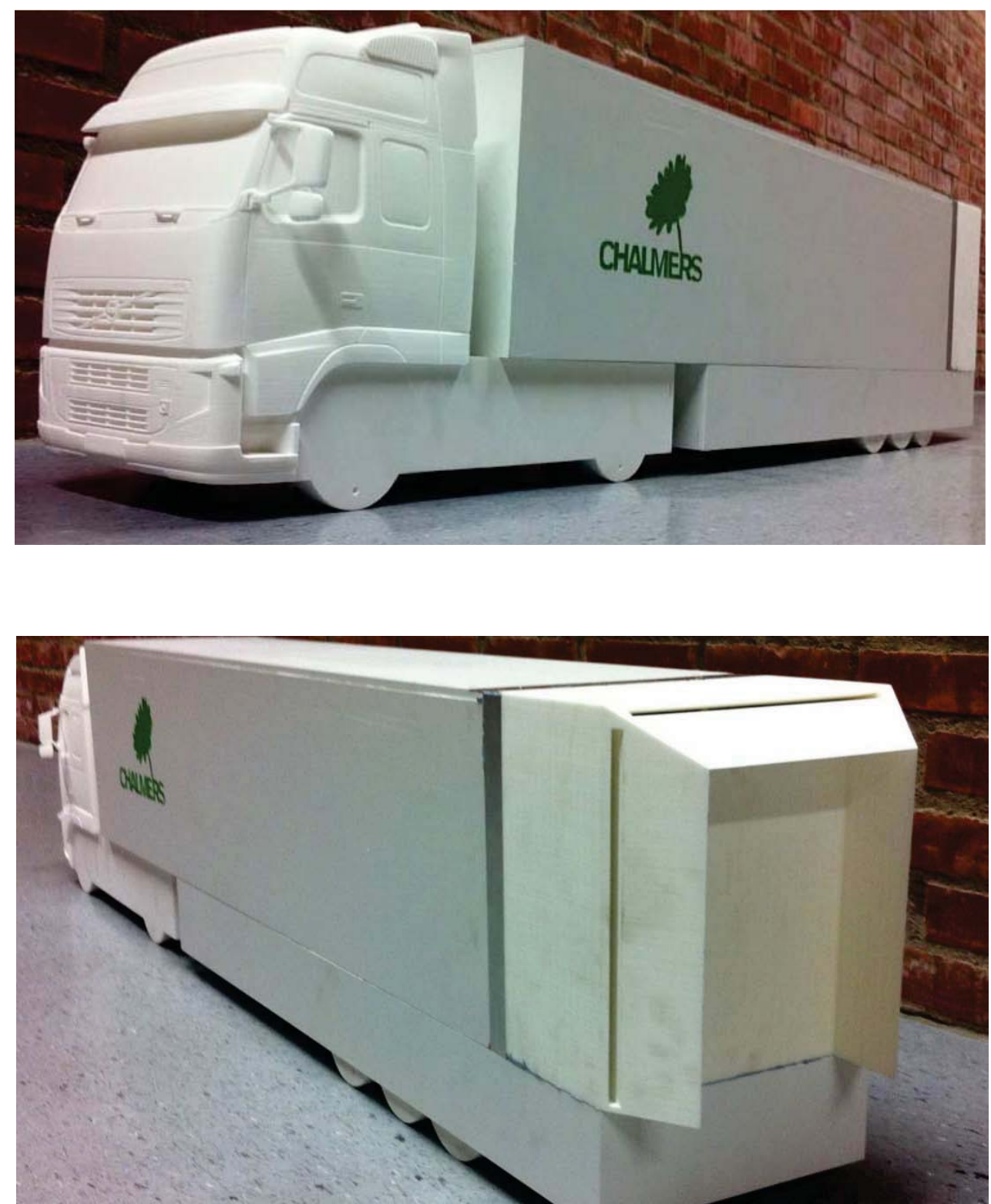

Figure 1: A front and rear view of a 1:10 experimental scale model of a VOLVO FH16 with flaps. The slots are also shown in the rear view.

In the present study the experiments and computations were performed for exactly the same model geometry and flow parameters. The angle of the flaps was 30 degrees and the flow angle from the actuator slot was 45 degrees relative to the flap surface. These parameters were found to be optimal in previous work [7-9]. The optimal actuation frequency was also selected on the basis of previous studies. The optimal actuation frequency and amplitude were also validated in the current experiments by varying these parameters and monitoring the drag reduction. 

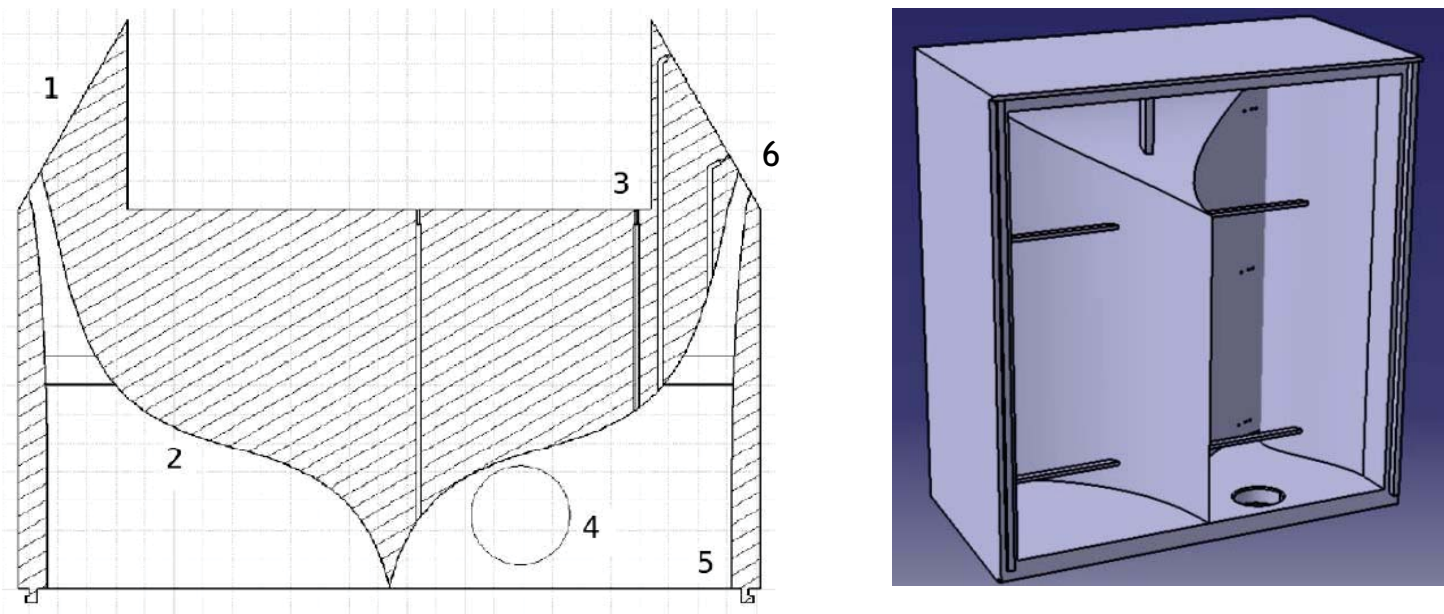

Figure 2: A cross section and 3D figure of the interchange rear end modules. In the cross section (top-view) we can see the flaps (1), the profile channels that guide the air from loudspeaker to the slot on the flap surface (2), the integrated pressure channels in the geometry (3), the exit of the pressure tubes (4) the profile of the rails that connect this module with the trailer (5) and finally the AFC slots (6).

a)

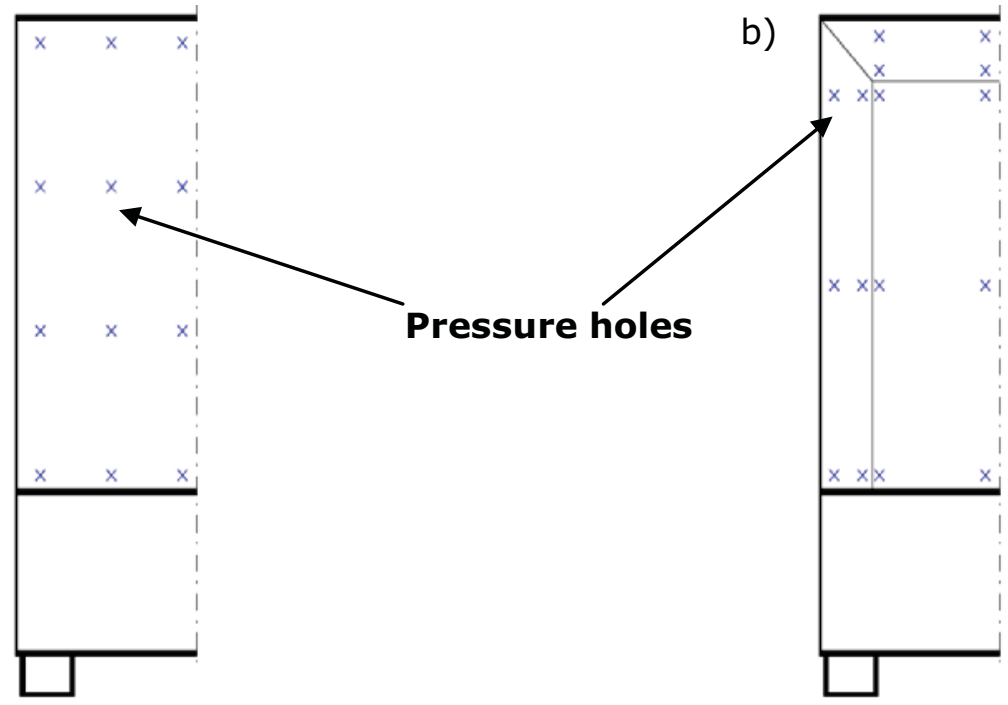

Figure 3: The locations of the pressure holes for the baseline (a) and PFC/AFC (b) cases. 


\section{EXPERIMENTAL DETAILS}

Experiments were carried out in a closed circuit wind tunnel with test section dimensions of $3.00 \times 1.80 \times 1.25 \mathrm{~m}^{3}$ and a speed range of $0-60 \mathrm{~m} / \mathrm{s}$, see Figure 4 . The actuator used in the experiments was a synthetic jet actuator based on a loud speaker enclosed in cavity. The flow actuation was performed through the slots located at the corner of the rear end and flaps. The loud speaker was mounted inside the trailer and the flow was guided via the profiled channels (Figure 2) in the trailer to the slots at the rear end. During experiments, the velocity from the actuator slot was measured by traversing a hot-wire probe at the slot outlet. The drag force of the model was measured using an external sixcomponent balance. The base pressure is monitored by a number of pressure taps positioned on the surface of the trailer base and flaps (Figure 3). The visualization of the flow separation was performed by surface tufts. The flow Reynolds number was 250,000 in the main series of experiments and a Reynolds number sweep was from 80,000 to 500,000 in order to measure the $C_{D}$ dependency on the Reynolds number.

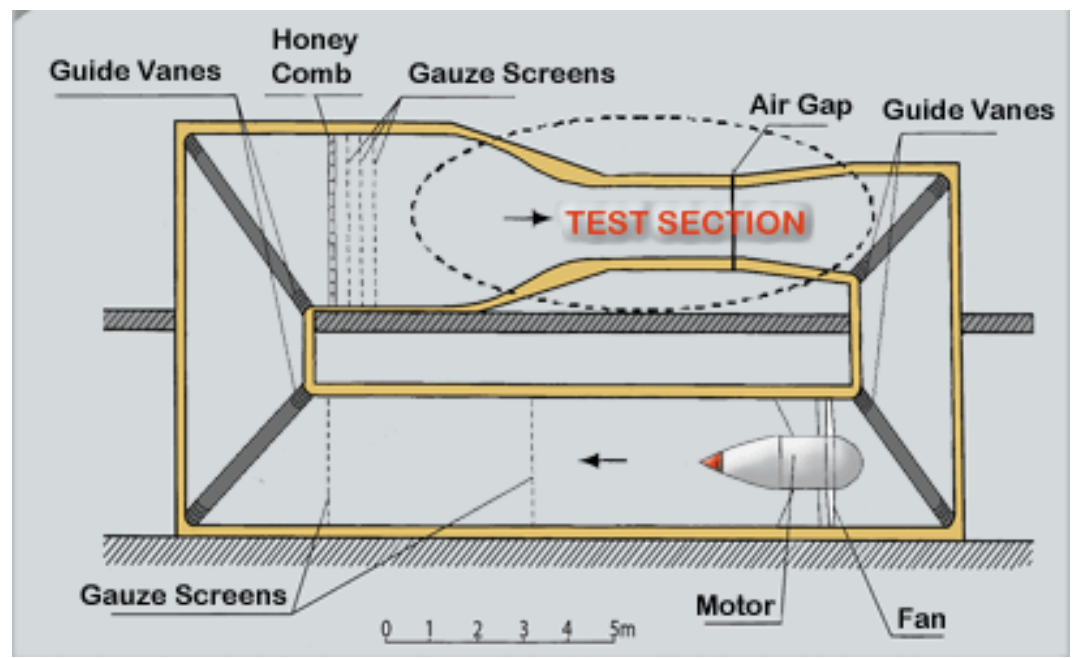

Figure 4: The experiment was carried out in Chalmers close circuit wind tunnel.

\section{Numerical Details}

The numerical simulations were performed by STAR-CCM+, a widely used commercial CFD software in the automotive industry (including Volvo 3P). Detached-eddy simulations with $k-\omega$ SST turbulence model were adopted. This model was chosen for several reasons. First of all, the case is transient because the actuation is sinusoidal blowing and suction. The geometry is complicated. URANS does not capture the details of the wake structure, which are important when simulating AFC/PFC. the DES was therefore an obvious choice for this type of flow and geometry. DES uses RANS for the boundary layer and LES for the detached eddies. The choice of RANS model is important. The DES based one-equation models use the distance to the walls as a length scale in RANS regions, which is not relevant in many flows, such as the complex external boundary layer flow of trucks and close to actuated regions, as in this case. A DES based on two-equation models uses either two turbulent quantities to obtain the turbulent length scale, e.g. $\mathrm{k}^{1 / 2} / \omega$, or the filter width delta. When the grid is sufficiently fine, the turbulent length scale switches from a RANS scale to a LES scale. 
The choice of the SST (Shear Stress Transport) model is motivated by superior treatment of the boundary layer as compared to a simple $k-\varepsilon$ or $k-\omega$ model. The SST is a combination of a $k-\omega$ and $k-\varepsilon$ in the inner boundary layer (BL) and outer $\mathrm{BL}$, respectively, and limits the shear stress in adverse pressure gradient regions. The dissipation term in

$$
\begin{aligned}
\beta^{*} k \omega & \rightarrow \beta^{*} k \omega F_{D E S}, \quad F_{D E S}=\max \left\{\frac{L_{t}}{C_{D E S} \Delta}, 1\right\} \\
\Delta & =\max \left\{\Delta x_{1}, \Delta x_{2}, \Delta x_{3}\right\}, \quad L_{t}=\frac{k^{1 / 2}}{\beta^{*} \omega}
\end{aligned}
$$

the $k$ equation is modified in order to get the DES, which switches the turbulent length scale from RANS to LES. Following [11], this is done according to

where $\beta^{*}=c_{\mu}$ and $\mathrm{F}_{\mathrm{DES}}$ is the damping function. The same truck geometry as in experiments was used and the computational domain size was adopted as the wind tunnel size. The active flow control was implemented as a sinusoidal time-varying boundary condition prescribed on the surface of the slot. The computational meshes consist of 30-40 million hexahedral cells with several refinement regions close to the surface of the model and flap region. The near-wall region is well resolved with $\mathrm{y}^{+}$below 1 almost everywhere.
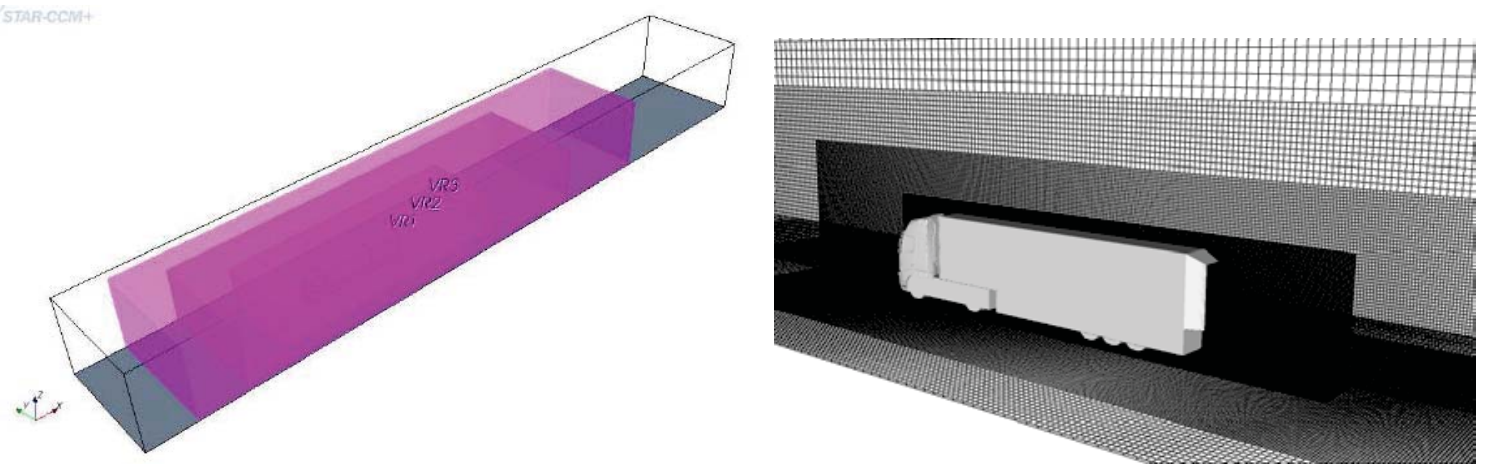

Figure 5: The computational domain with refinement regions and a slice in the center of the computational mesh.

\section{Results AND Discussion}

\subsection{Experiment}

To ensure $R e$ number independent drag results, a $R e$ number sweep was carried out between $R e=80,000$ and 500,000 , which corresponds to $U=5-30 \mathrm{~m} / \mathrm{s}$. The sweep was carried out both for the baseline and the PFC case. It was difficult to do this for the AFC case because of the frequency dependent results. Figure 6(a) plots the drag versus $R e$ for both cases and shows that the drag is stabilized for $R e>160,000$, which corresponds to a velocity of $10 \mathrm{~m} / \mathrm{s}$ in the wind tunnel. The velocity chosen was $15 \mathrm{~m} / \mathrm{s}$ $(R e=250,000)$. Several measurements of the actuator with different amplitudes and frequencies were carried out to check the frequency dependence of the actuator, and these are plotted in Figure 6(b). It is clear that the performance of the actuator is frequency dependent. We found that a frequency of $100 \mathrm{~Hz}$ gives a maximum output velocity from the actuator. Based on Strouhal number equivalency, the frequency should be $100 \mathrm{~Hz}$. 
a)

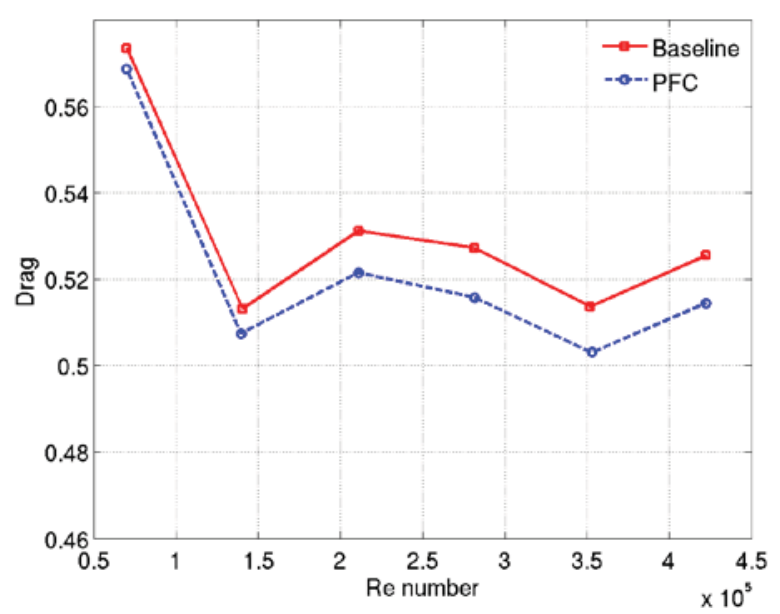

b)

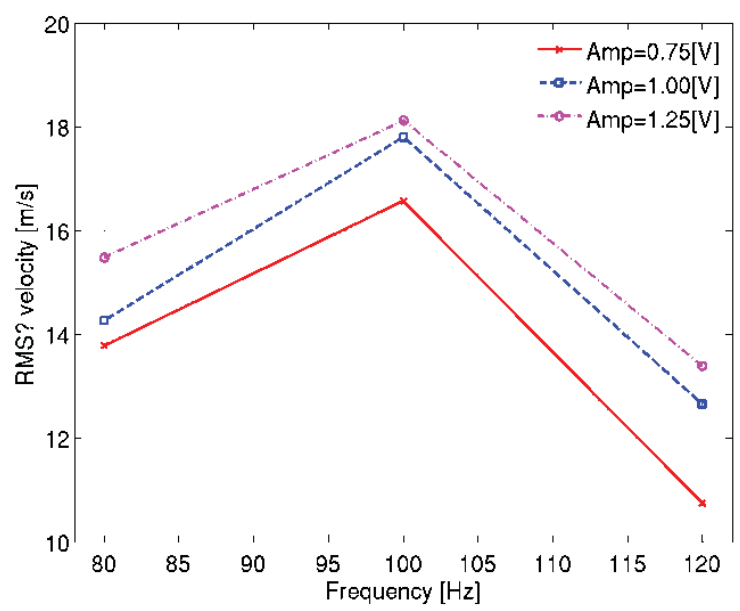

Figure 6: A Re sweep (a) to ensure Re independent drag for both the baseline and the PFC case. The frequency response (b) of the implemented actuator.

The forces measured for the different configurations are presented in Table 1 . The drag coefficient value was computed using the projected frontal area, numerically found in Star-CCM+. The air density was calculated from equation of state and the wind tunnel velocity was reported during the measurements. The parameter values for the AFC case are $f=100 \mathrm{~Hz}$ and amplitude $A=1 \mathrm{~V}$ of the actuator sinusoidal slot velocity. Figure $7(\mathrm{a})$ plots the drag change for different AFC frequencies and amplitudes. It can be concluded that It is not necessary to have a very high velocity output from the actuator in order to have the highest drag reduction. The output from the actuator is highly frequency dependent. For two amplitudes, $0.75 \mathrm{~V}$ and $1.0 \mathrm{~V}$, the drag decreased with frequency. This was not true however for actuator amplitude of 1.25 . Nonetheless, the results show an overall drag reduction by both the PFC and the AFC configuration.

\begin{tabular}{l|c|c} 
Configuration & $C_{D}$ & Delta $C_{D}[\%]$ \\
\hline Baseline & 0.53 & - \\
\hline PFC & 0.52 & -1.77 \\
\hline AFC & 0.51 & -3.13
\end{tabular}

Table 1: Experimental drag values of different configurations.

The base pressure was also measured, as mentioned in section 2. Table 2 reports the area-averaged base pressure for different configurations. The parameter values for the AFC case, as mentioned earlier, were $f=100 \mathrm{~Hz}$ and $A=1 \mathrm{~V}$. Figure $7(\mathrm{~b})$ depicts the base drag change for different AFC frequencies and amplitudes. The base pressure coefficient is defined as $C_{p t r}=\left(p-p_{\infty}\right) /\left(1 / 2 \rho V^{2}\right)$. The results show that the base pressure increases a great deal with AFC, as expected. The drag reduction (Figure 7(a)) is however smaller than the reduction in base pressure. The increase in base pressure is also frequency dependent, and it can be concluded from Figure 7(b) that a frequency increase up to 100 $\mathrm{Hz}$ is favorable. It is also noticeable that the drag reduction (Figure $7(\mathrm{a})$ ) and base pressure increase (Figure $7(b)$ ) do not correlate as expected. Only the AFC amplitude of $0.75 \mathrm{~V}$ correlates well, but the amplitude of $1.25 \mathrm{~V}$ at $100 \mathrm{~Hz}$ has the highest increase in base pressure but with the smallest reduction in drag. It is conjectured that the output 
velocity of the actuator is highest at this frequency and also with maximum amplitude, the high velocity may act as a blockage to the main stream, which causes the separation at the trailing edge to occur earlier.

\begin{tabular}{l|l|c} 
Configuration & $\boldsymbol{C}_{\boldsymbol{p t r}}$ & $\boldsymbol{\Delta} \boldsymbol{C}_{\boldsymbol{p t r}}[\mathbf{\%}]$ \\
\hline Baseline & -0.28 & - \\
\hline PFC & -0.22 & -21.4 \\
\hline AFC & -0.17 & -39.3
\end{tabular}

Table 2: Area-averaged base pressures of the different configurations.

a)

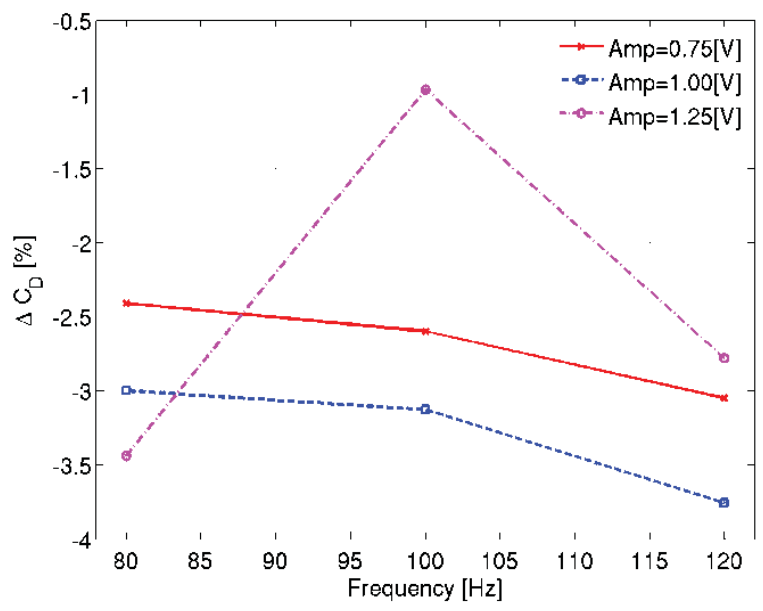

b)

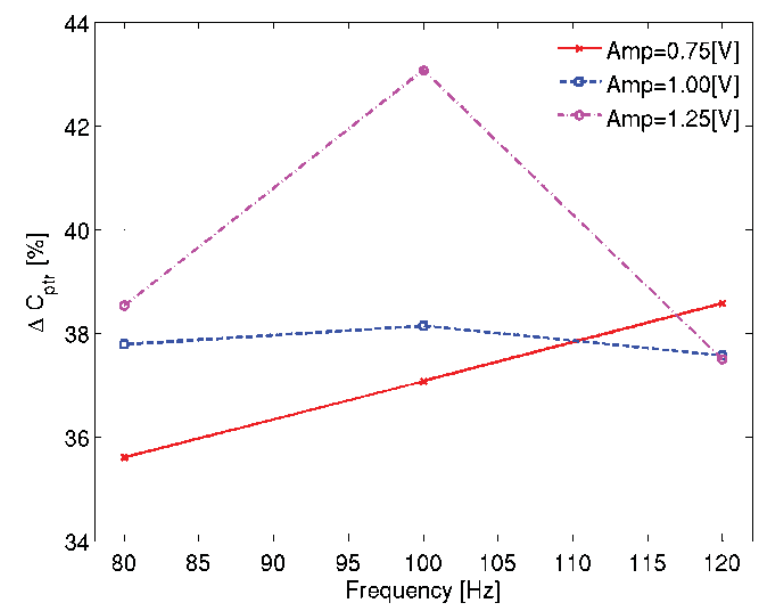

Figure 7: Drag change (a) and area-averaged base drag (b) due to different frequencies and amplitudes of the implemented actuator. Reduction in percent relative to baseline case.

Figure 8 plots the distribution of the base pressure at one half side of the trailer (the flow is symmetric). These plots show where the base pressure increases locally with PFC and AFC. The major increase in base pressure is in the upper half of the trailer. The lower half is affected but not as much as the upper one. The reason is that there is no flap on the lower side of the trailer. This is due to practical reasons for the use of trailers in the cargo industry and because the incoming underbody flow is difficult to control. The effect of the actuation is evident in Figure 8(c); a low pressure region is created along the trailer sides. The AFC mechanism creates a low pressure region that reattaches the separated flow on the flap surface. This effect is visualized in the wind tunnel using tufts which are distributed at different locations on the rear end of the trailer. Figure 9 depicts the difference between the PFC and AFC flow field. The tufts at the trailing edge of the trailer for the PFC case (Figure 9(a)) are non-uniformly oriented. This is due to the separated flow region. During the experiment, the tufts at the flap trailing edge were also randomly moving, which showed the effect of the strong vortex shedding. The AFC case in Figure 9(b) has streamlined tufts at all places. This shows that the AFC evidently succeeded in reattaching the separated flow. During the experiment it was noticed that the flow reattached immediately when AFC was set on. 
a)

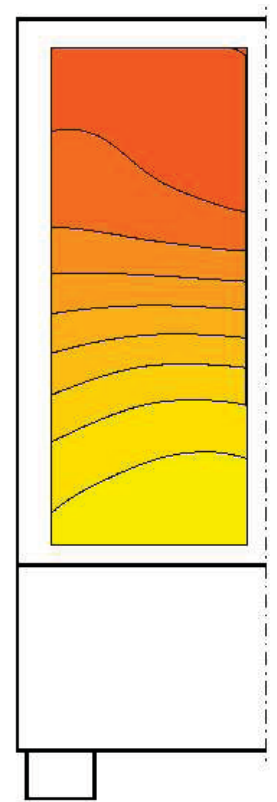

b)

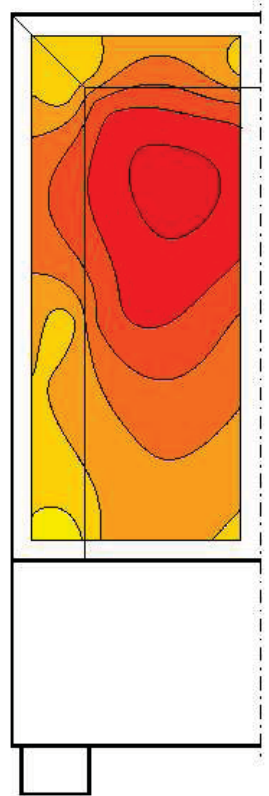

c)

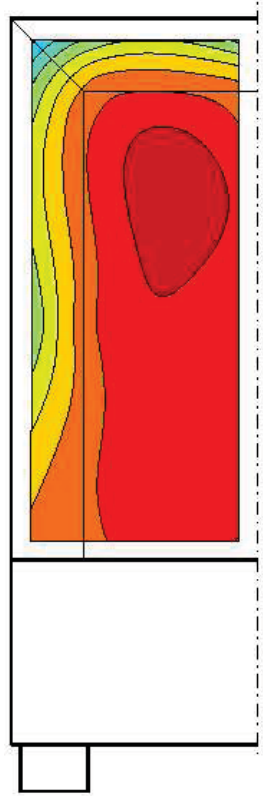

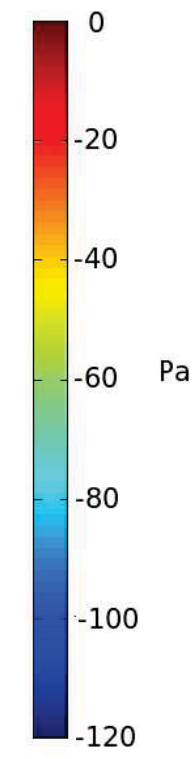

$\mathrm{Pa}$

Figure 8: Experimental base pressure distribution for baseline (a), PFC (b) and AFC (c) configuration.

a)

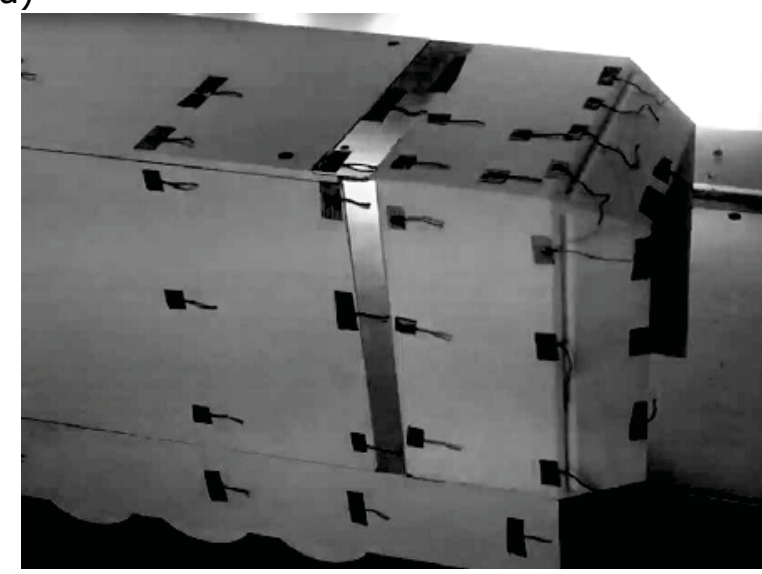

b)

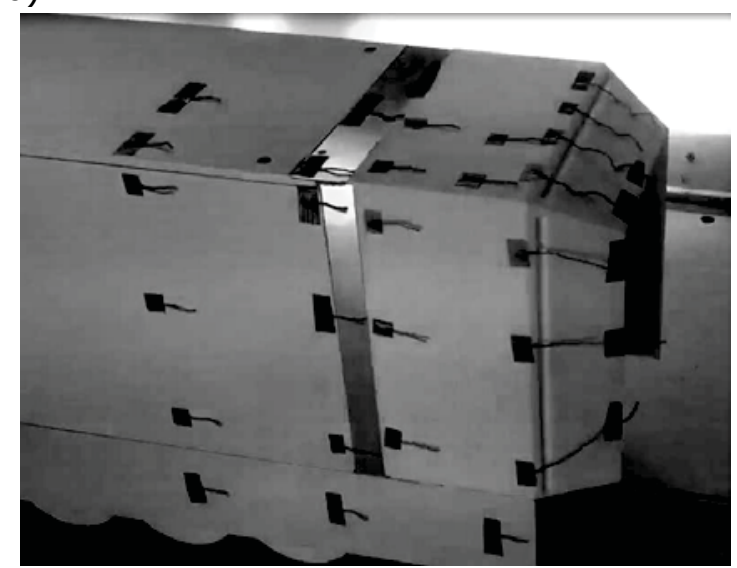

Figure 9: Flow visualization by tufts in the wind tunnel, PFC (a) and AFC (b).

\subsection{Computations}

Three configurations were numerically simulated: baseline, PFC and AFC. The configurations are the same as the experimental set-up except that for the AFC case, a lower output velocity from the actuator is used because large numerical fluctuations were encountered. The objective of these simulations was to check whether a reduction in drag is obtained with PFC or AFC. The simulations were very challenging owing to convergence issues. The numerical procedure is first to run each case in steady state using RANS with a first-order precision scheme followed by a second-order scheme and finally to run the case with DES. The AFC case is restarted from a converged solution of the PFC case. The time-averaged drag values are found in Table 3, where the 
experimental values are also repeated for comparison. The trends are captured by DES, especially the reduction in drag for the AFC case. The drag history is plotted in Figure 10. In Figure 10(b) we observe the high amplitude fluctuations caused by the actuation boundary condition. Efforts were made to minimize the fluctuations but it seems that this is an unsolved issue.

Only the PFC and AFC cases are compared because the baseline and PFC cases have quite similar results.

\begin{tabular}{|c|c|c|c|c|}
\hline Configuration & $\begin{array}{l}C_{D} \\
\text { Exp }\end{array}$ & $\begin{array}{c}\Delta C_{D} \\
\operatorname{Exp}[\%]\end{array}$ & $\begin{array}{c}C_{D} \\
\text { DES }\end{array}$ & $\begin{array}{c}\Delta \mathrm{C}_{\mathrm{D}} \\
\text { DES [\%] }\end{array}$ \\
\hline Baseline & 0.53 & - & 0.55 & - \\
\hline PFC & 0.52 & -1.77 & 0.54 & -0.66 \\
\hline AFC & 0.51 & -3.13 & 0.53 & -3.96 \\
\hline
\end{tabular}

Table 3: Experimental and numerical drag values of different configurations.

a)

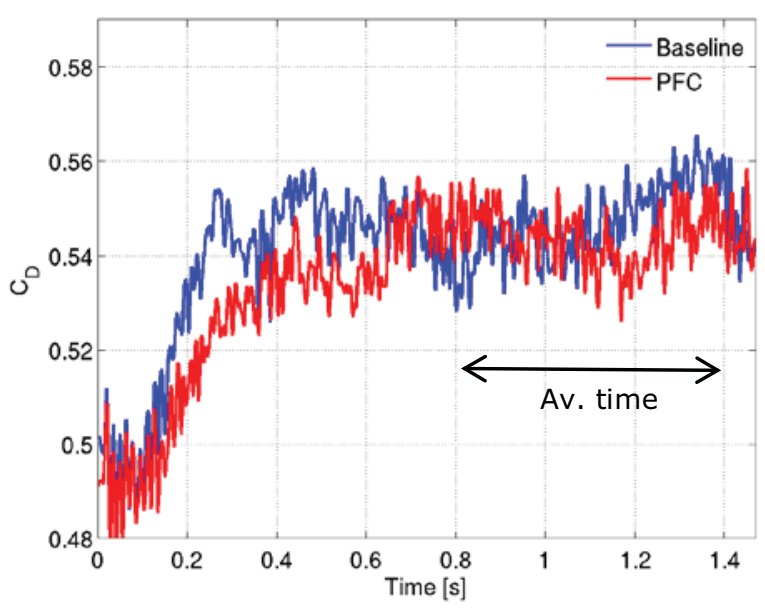

b)

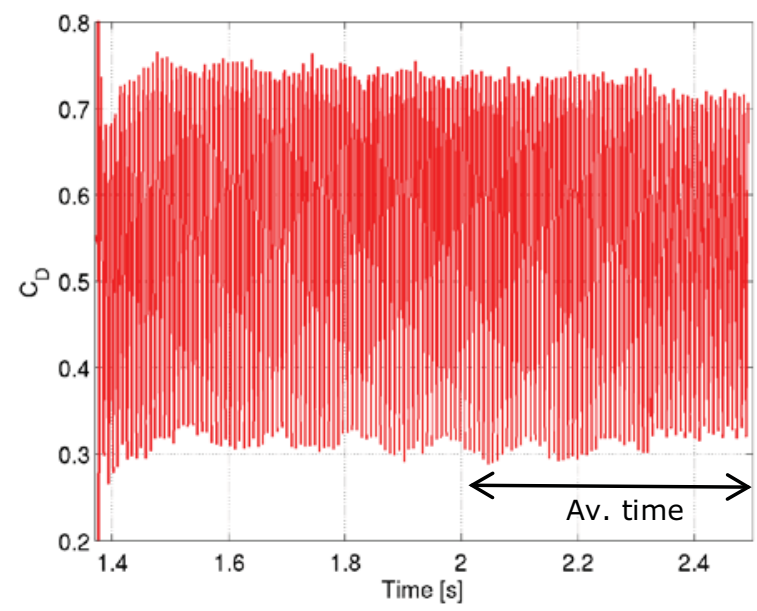

Figure 10: Drag history of baseline, PFC (a) and AFC (b) configurations.

Figure 11 depicts an overall flow field view of the PFC and AFC configurations. One can notice the highly non-uniform side flow and the front separation region on the upper leading edge of the truck, which affects the downstream flow. The strong wake of the PFC case can be observed in Figure 11(a), which is caused by the separated flow on the flap. The wake size is narrowed and the separation at the flap is delayed in the AFC case (Figure 11(b)). The reattached flow on the flap surface reduces the size of the wake. The base pressures for the PFC and AFC case are plotted in Figure 12. There is a great pressure recovery at the rear end of the trailer in the AFC case. These numerical results are in agreement with the experimental ones in Figure 8. The separated flow reattaches and the base pressure consequently increases which gives a drag reduction. The effect of the actuation can also be noticed by the low pressure region at the upstream part of the flaps in Figure 12(b). This is due to the high sinusoidal fluctuating velocity component from the actuator slots along the flaps. 
a)

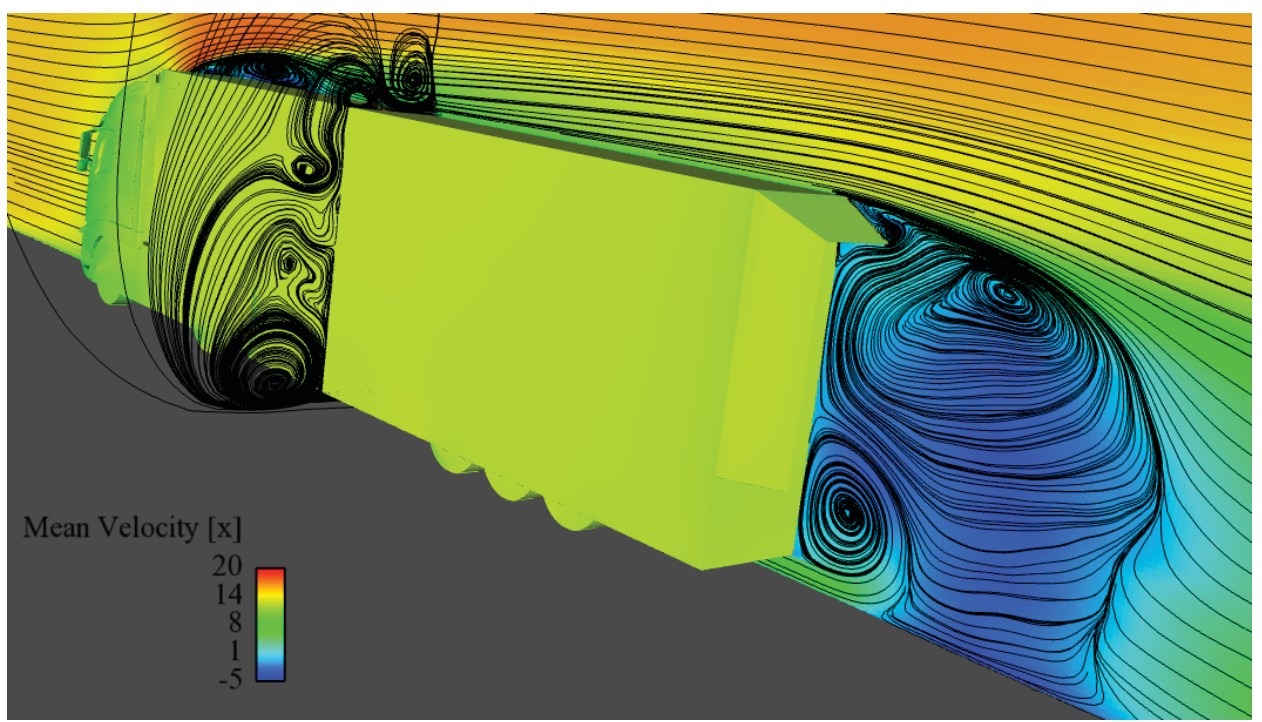

b)

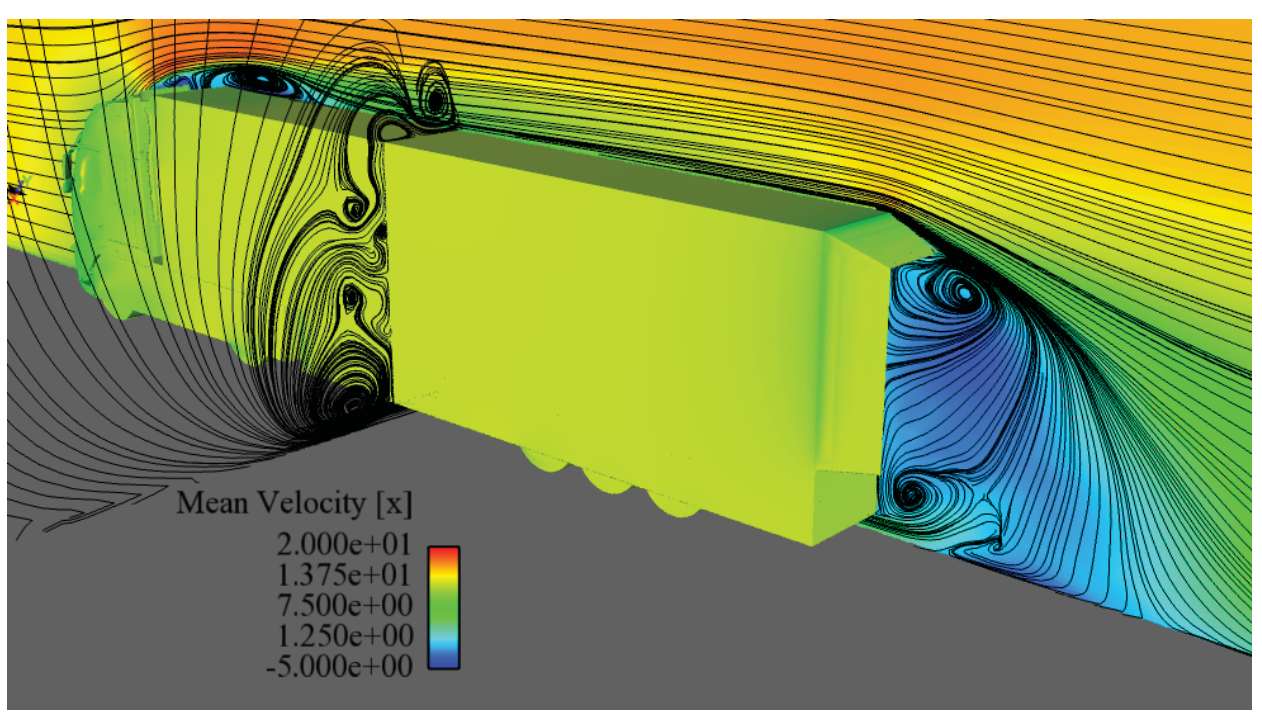

Figure 11: Time-averaged streamlines and velocity contours of the truck with trailer: PFC (a) and AFC (b) configurations.

Figure 13 depicts the contours of time-averaged pressures in the $x-y$ plane which shows a part of the computational domain close to the truck-trailer and a zoom in the wake region. There is a great pressure recovery in the wake of the AFC configuration (Figures 13 (c) and (d)) compared with the PFC one (Figures 13 (a) and (b)). A large lowpressure region along the front side of the truck is shown. This is due to the separated flow in this region. The actuation effect is also shown in Figure $13(d)$ as a large lowpressure region at the flap corner. This effect may be one of the reasons that the separated flow reattaches on the flap surface. 
a)

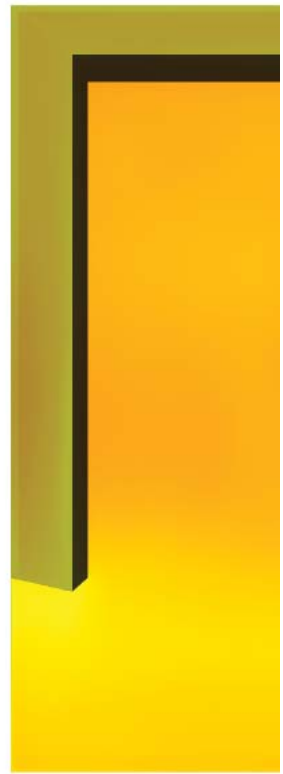

b)

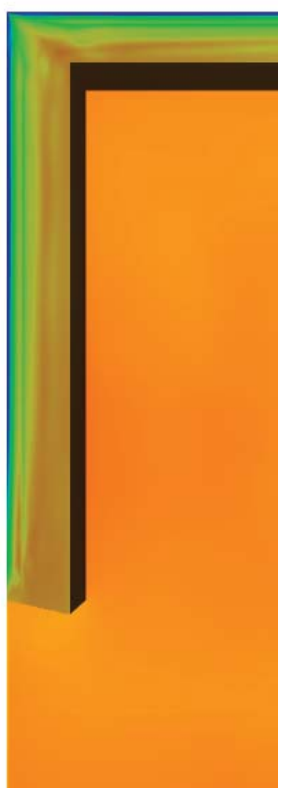

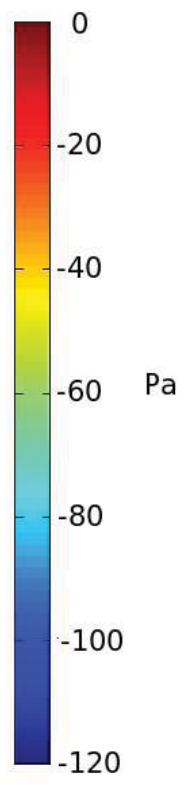

Figure 12: Numerical base pressure distribution of PFC (a) and AFC (b) configurations.

Figure 14 depicts the turbulent kinetic energy in the wake behind the trailer. The turbulence is enhanced along the flap surface in the AFC case (Figure 14(b)) compared to the PFC case (Figure 14(a)). There is also a slight increase in turbulence level in the wake of the AFC case. The latter is a consequence of the reattached flow and also demonstrates an increase of flow activity in the wake.

Figures 15 and 16 show the streamlines in different views. These also confirm the narrower and shorter wake size of the AFC configuration (Figure 15(b)) compared to the PFC one (Figure 15(a)). It is noticeable that the lower dead region in the wake of the PFC configuration (Figures 16(a) and 16(c)) is alleviated by the AFC (Figures 16(a) and 16(c)). 
a)

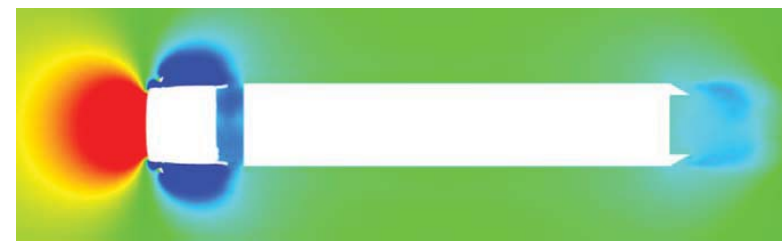

b)

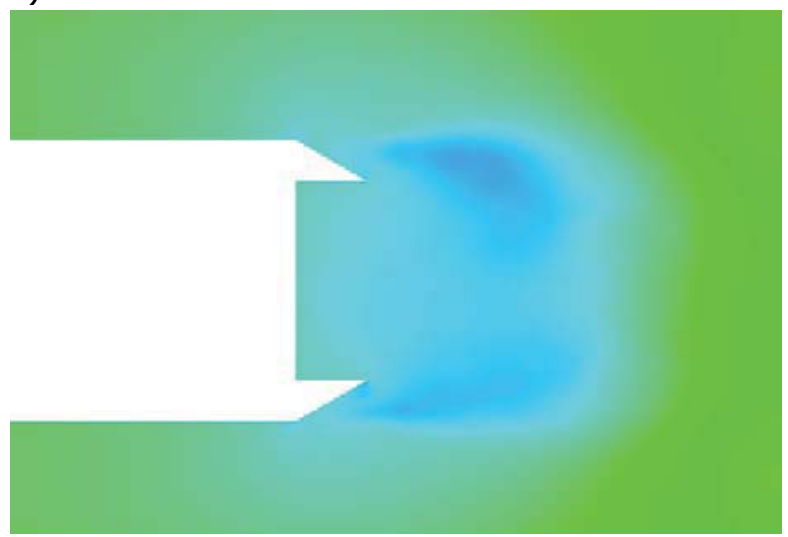

c)

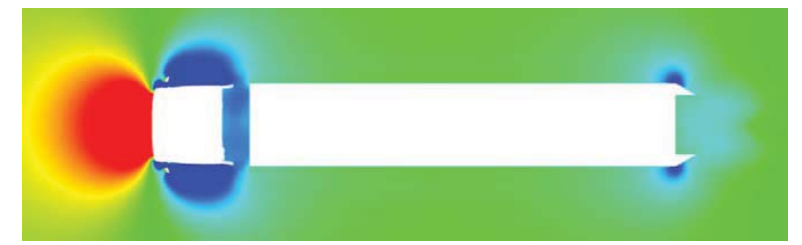

d)

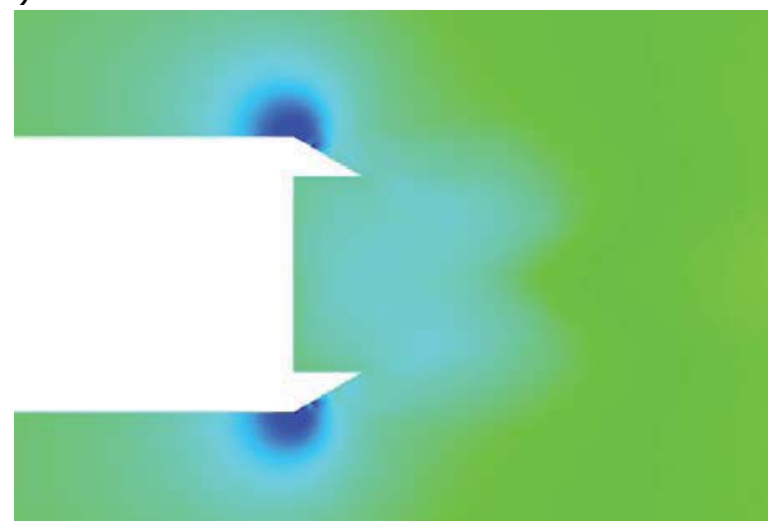

Figure 13: Contours of time-averaged pressure in the wake region (at $50 \%$ height): PFC full truck (a), PFC wake region (b), AFC full truck (c) and AFC wake region (d).

a)

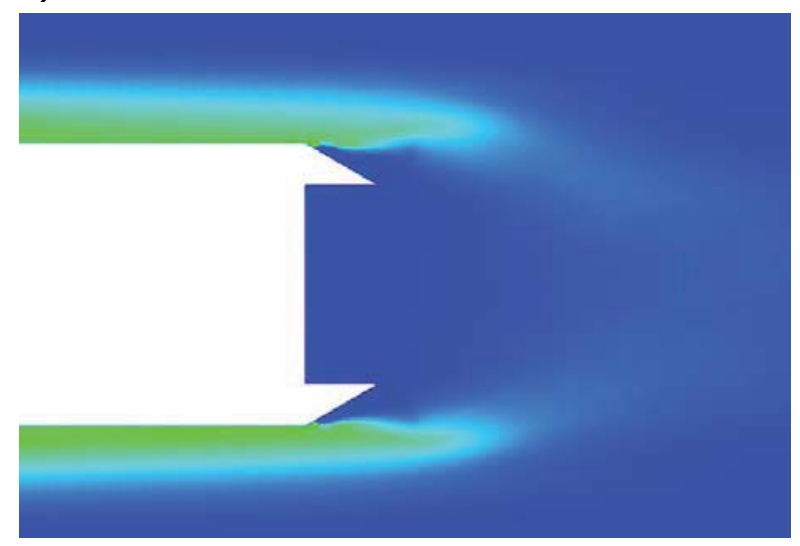

b)

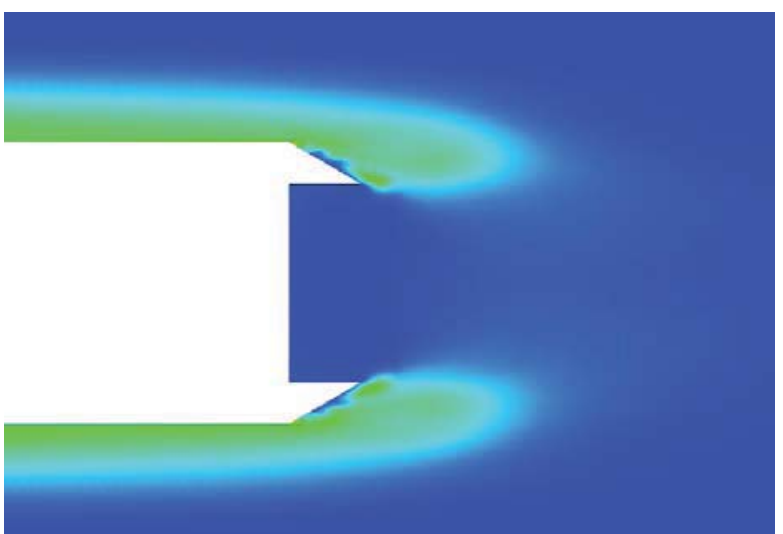

Figure 14: Contours of time-averaged turbulent kinetic energy in the wake region (at $50 \%$ height): PFC (a) and AFC (b) configurations. 
a)

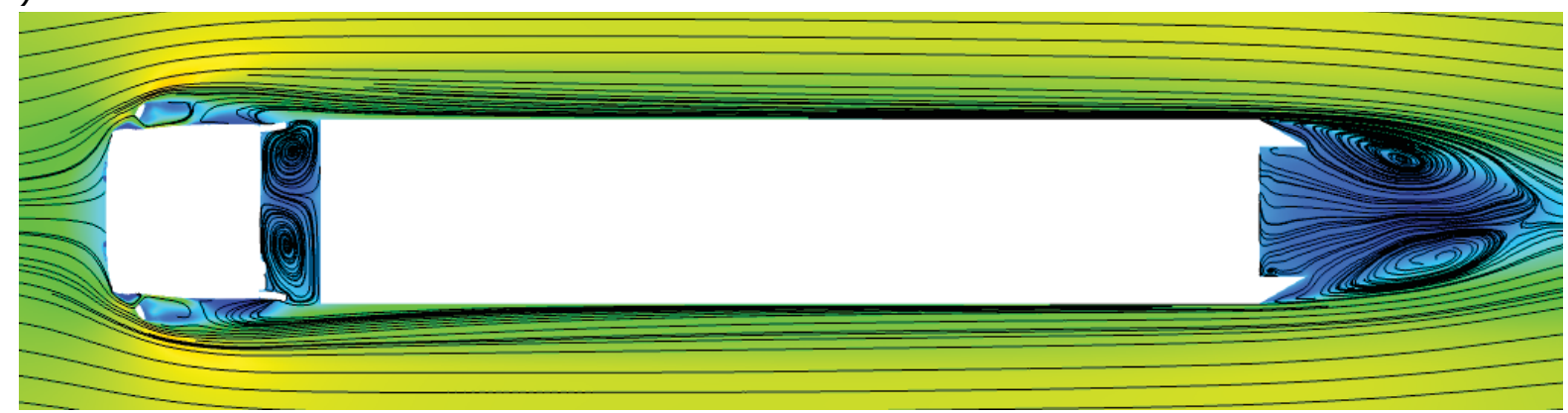

b)

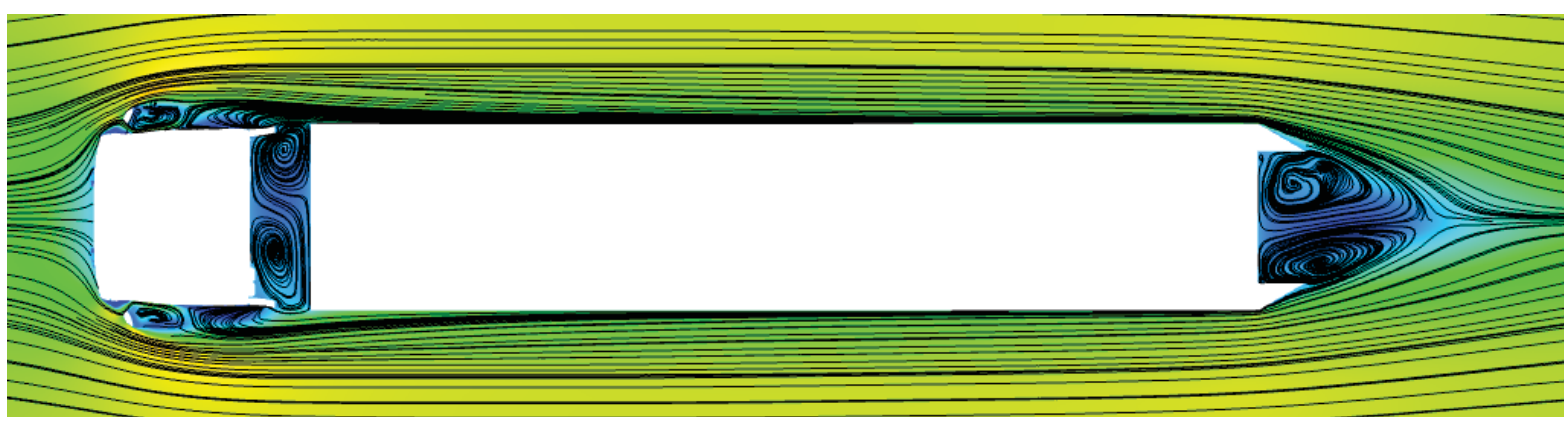

Figure 15: Contours of time-averaged $x$-velocity and streamlines $x-z$ view (at $50 \%$ height): PFC (a) and AFC (b) configurations.

a)

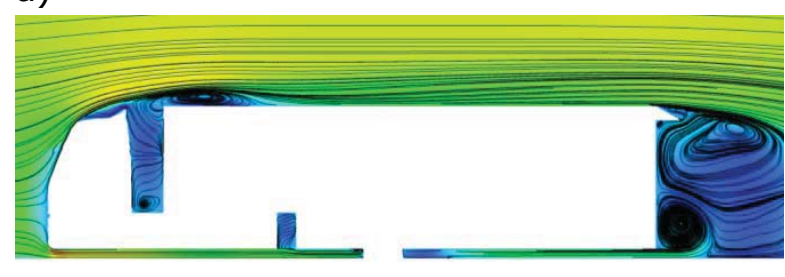

c)

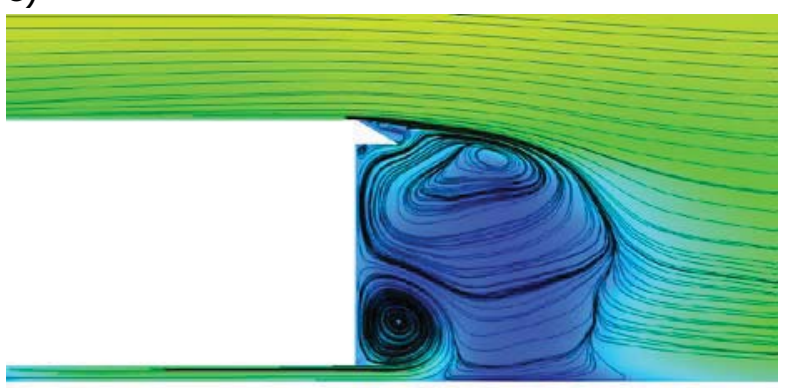

b)

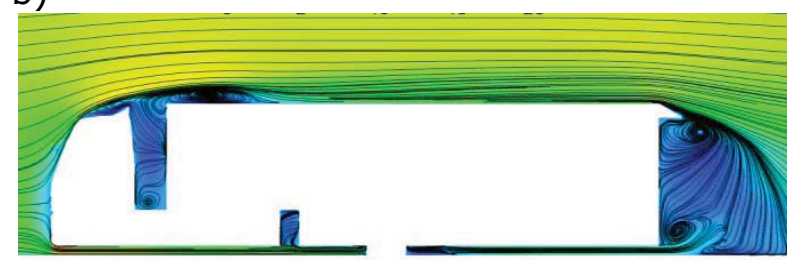

d)

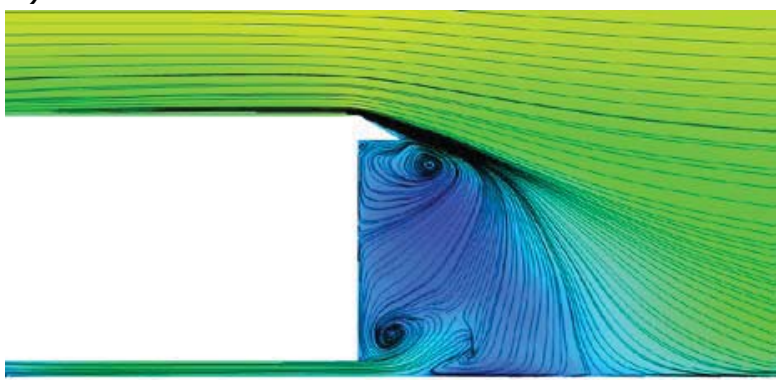

Figure 16: Contours of time-averaged $x$-velocity and streamlines $x-z$ view (symmetry plane): PFC (a) and (c) and AFC (b) and (d) configurations. 


\section{Conclusions}

Both experiments and computations demonstrate that the active flow control works successfully and results in the flow attaching to the flaps. The numerical simulations show that the drag coefficient, $C_{D}$, decreased by $6.8 \%$ when AFC is activated compared to the baseline case without flaps. The corresponding decrease when AFC is deactivated is only $1.2 \%$. The experimental results show a decrease in $C_{D}$ of $3.1 \%$ for the case with AFC activated compared to the baseline case. With AFC deactivated, the corresponding decrease in $C_{D}$ was $1.8 \%$. Detailed flow analysis made in computations and experiments is used to explain these results.

\section{ACKNOWLEDGEMENTS}

This work is supported by the Swedish Agency of Innovation Systems (VINNOVA), Volvo 3p, SKAB and CD-ADAPCO. The financial support of SNIC (the Swedish National Infrastructure for Computing) for computer time at C3SE (Chalmers Centre for Computational Science and Engineering) is gratefully acknowledged. Special thanks to Fred Ross at CD-ADAPCO for providing valuable guidlines for DES simulations in STAR$\mathrm{CCM}+$. Linus Hjelm, Zenitha Chronéer and Jan Niklasson at Volvo 3P provided valuable contributions to this project, including CAD model and printing the model in the SLS machine. Jan Bragée and Nils Nilsson from the prototyping laboratory at Chalmers University of Technology provided important theoretical and practical advices in the construction work of the truck model.

\section{REFERENCES}

[1] Prandtl, L.,: Uber Flussigkeitsbewegung bei sehr kleiner Reibung In: Proceedings of the third international mathematics congress. Heidelberg; pp. 48491, 1904.

[2] Lachman G, editor., :Boundary Layer and Flow Control vols. 1, 2. Oxford: Pergamon Press; 1961.

[3] Gad-el-Hak, M: Introduction to flow control In: Gad-el-Hak M, Pollard A, Bonnet J, editors. Flow control: Fundamentals and Practices. Berlin: Springer, pp. 199-273, 1998.

[4] Gad-el-Hak, M., Pollard A, Bonnet J, editors: Flow control: fundamentals and practices, Berlin: Springer, 1998.

[5] Schubauer, G. B., Skramstad, H. K.: Laminar boundary layer oscillations and transitions on a flat plate, J Aero Sci;14, pp. 69-79, 1947.

[6] Seifert, A., Darabi, A., and Wygnanski: I. Delay of airfoil stall by periodic excitation, Journal of Aircraft, Vol. 33, No. 4, pp. 691-699, 1996.

[7] El-Alti M.: Active Flow Control for Aircrafts and Heavy Vehicles: Thesis for licentiate of engineering 2009:011, Div. of Fluid Dynamics, Dept. of Applied Mechanics, Chalmers University of Technology, Göteborg, Sweden, 2009.

[8] El-Alti M., Kjellgren P., Davidson L.: Drag reduction for trucks by active flow control of the wake behind the trailer. In: 6th International Symposium on Turbulence, Heat and Mass Transfer, 2009.

[9] El-Alti M., Chernoray V., Kjellgren P., Hjelm L., Davidson L.: Computations and full-scale tests of active flow control applied on a VOLVO truck-trailer, In Aerodynamics of Heavy Vehicles III: Trucks, Buses and Trains, 2010. 
[10] Andersson E., Andersson E., Granström B., Löfgren N., Nyberg N., Svedman J.: Aktiv strömningskontroll av vaken bakom en lastbil: Ett försök att minska bränsleförbrukningen hos lastbilar, Kandidatarbete 2011:03, Div. of Fluid Dynamics, Dep. of Applied Mechanics, Chalmers University of Technology, Göteborg, Sweden, 2011.

[11] Davidson L.: Fluid Mechanics, turbulent flow and turbulence modeling, Div. of Fluid Dynamics, Dep. of Applied Mechanics, Chalmers University of Technology, Göteborg, Sweden, 2011. 